\title{
The critical role of FDG-PET/CT imaging in assessing systemic manifestations of COVID-19 infection
}

\author{
Abass Alavi $^{1}$ - Thomas J. Werner ${ }^{1}$ - Ali Gholamrezanezhad ${ }^{2}$ \\ Published online: 8 January 2021 \\ (C) The Author(s), under exclusive licence to Springer-Verlag GmbH, DE part of Springer Nature 2021
}

Coronavirus disease 2019 (COVID-19) is an infectious disease that primarily involves the lungs with associated respiratory syndrome but may also demonstrate extrapulmonary/ systemic manifestations throughout the body [1]. The clinical presentation of this disease includes fever, cough, shortness of breath, malaise, flu like body ache, abdominal pain, diarrhea, and loss of smell and taste [2]. The time from exposure to development of symptoms varies somewhat, but in general, it is between 2 days and 2 weeks. In addition to pulmonary symptoms and signs, COVID-19 can manifest with evidence of inflammation in other organs, including the bowel, liver, brain, kidneys, and the cardiovascular system [1]. These patients are also prone to developing cerebrovascular disease as well as deep vein thrombosis [3].

A variety of blood and imaging tests are performed for the diagnosis, evaluation of the extent, and the assessment of the associated complications of the disease. These include immunologic parameters that are the hallmark of this viral infection and are routinely performed in the affected or at-risk population. However, imaging techniques, including chest X-ray and $\mathrm{CT}$, have been typically employed for detecting and determining the extent of pulmonary involvement [4]. Chest CT typically demonstrates multifocal peripheral ground glass opacities with or without superimposed consolidations in a posterior and basilar predominant distribution [5]. Crazy paving pattern and increased interstitial septal thickening are less common manifestations of COVID-19 on chest CT. Pleural

This article is part of the Topical Collection on Infection and inflammation.

Abass Alavi

abass.alavi@pennmedicine.upenn.edu

1 Department of Radiology, University of Pennsylvania, 3400 Spruce St, Philadelphia, PA 19104, USA

2 Keck School of Medicine, University of Southern California (USC), Los Angeles, CA, USA effusion and mediastinal lymphadenopathy are not very common. Chest X-ray findings are less sensitive with bilateral peripheral and basilar predominant air space opacities (Fig. 1) [5]. Recently, point-of-care ultrasound (PUS) has been proposed as an alternative imaging modality for detection of inflammatory lesions in the lungs.

By now, it is well established that the role of structural techniques, such as CT, US, and radiography, is suboptimal, because of their low specificity in assessing many infectious and inflammatory pulmonary diseases and disorders [6-8]. These shortcomings are also applicable to examining patients with COVID-19 with these structural imaging modalities. Therefore, there is a dire need for a technique with higher sensitivity and specificity for assessing patients with this serious infection. Furthermore, the information provided by these techniques are qualitative in nature and lack quantitative results which are essential for the early detection as well as response assessment. Therefore, imaging modalities that are highly sensitive and quantify the degree of disease activity throughout the body are highly desirable and will provide the required means to monitor the course of this infection.

Over the past four decades, FDG-PET imaging has been extensively employed to assess a variety of organ diseases and disorders [9-11]. While the initial application of this technique dealt with brain disorders, during the past three decades, this modality has been extensively promoted for diagnosing and staging cancer, monitoring response to treatment and detecting recurrence in various malignancies [6]. Furthermore, it has been established that activated inflammatory cells in any setting are highly glycolytic and therefore their presence is readily detected by FDG-PET in various organs in the body. As such, activated inflammatory cells due to infection will show substantially enhanced glycolysis and, therefore, can be readily detected by FDG-PET imaging. By now, the role of FDG-PET imaging for detecting and characterizing various inflammatory disorders has been validated in many settings $[12,13]$. This approach has been shown to be of great value in managing patients with serious infections such as TB and 


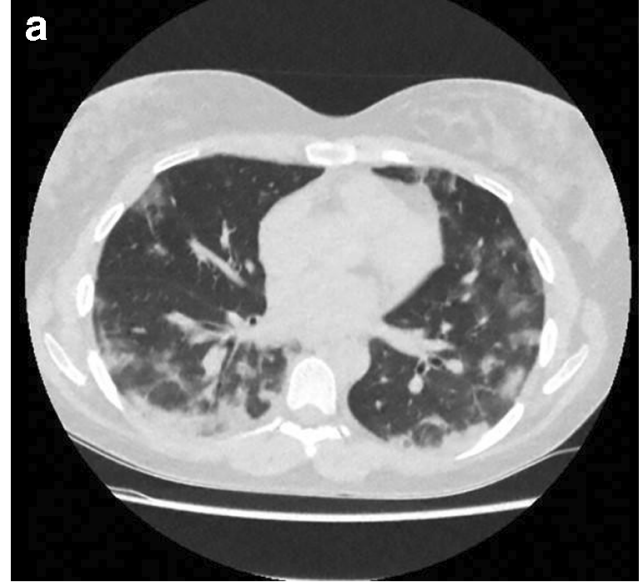

Fig. 1 Axial CT slices (lung window) in two patients with coronavirus disease 2019 (COVID-19) Reporting and Data System category 5 observations by all eight observers. a A 30 -year-old woman with reverse transcription-polymerase chain reaction test (RT-PCR) positive for severe acute respiratory syndrome coronavirus 2 who presented with fever and cough of 12 days duration. She was discharged after 7 days of inpatient

HIV-AIDS. Inflammatory reactions due to noninfectious etiologies have also been studied extensively with this technology with great success [14].

FDG-PET imaging can be used to determine the systemic effects of COVID-19 infection on the entire body (Fig. 2) [15]. Furthermore, systemic inflammatory response to COVID-19 infection is known to cause significant damage to the brain, the lungs, and the bowel while also causing arterial and venous thrombosis [1, 15-17]. In recent years, the introduction of modern PET imaging instruments which allow examining the entire body with a single data acquisition is ideally suited for examining patients with SARS-CoV-2

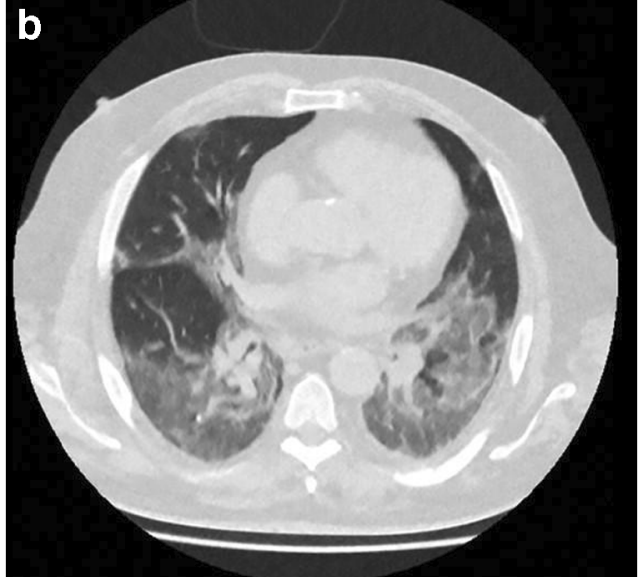

management, with resolution of symptoms. b A 51-year-old man presented after 8 days of fever, dyspnea, and cough. A clinical diagnosis of COVID-19 was made due to clinical symptoms and laboratory findings, despite negative results at repeated RT-PCR tests. This patient was admitted for 2 days due to hypoxia, with alleviation of symptoms after 5 days (reproduced with permission from Prokop $\mathrm{M}$ et al.) [5]

infection (Fig. 3) [18-21]. As noted above, this infection can potentially involve different body organs beyond the lungs and, therefore, imaging the whole body with this approach will be revolutionary in managing these patients.

Over the past decade, we and other investigators have employed FDG-PET to detect atherosclerosis and other inflammatory diseases of the major arteries in the body (Fig. 4) [22]. Furthermore, we have noted that venous clots due to their high concentration of activated white cells are also readily visualized by FDG-PET (Fig. 5) [23-27]. We believe this potential application of FDG-PET imaging will likely play a major role in evaluating and treating patients with
Fig. 2 a The initial PET/CT study reveals metabolically active lung infiltrates (black arrows), hypermetabolic hilar and mediastinal lymphadenopathy, and intense colonic activity (red arrows). b Follow-up PET/CT with resolution of lung and GI activity, leaving a metabolically active nodule that was obscured on the initial PET-CT by hypermetabolic COVID-19 pulmonary infiltrate (arrow). CT, computed tomography; GI, gastrointestinal; PET, positron emission tomography (reproduced with permission from Kidane B et al.) [15]

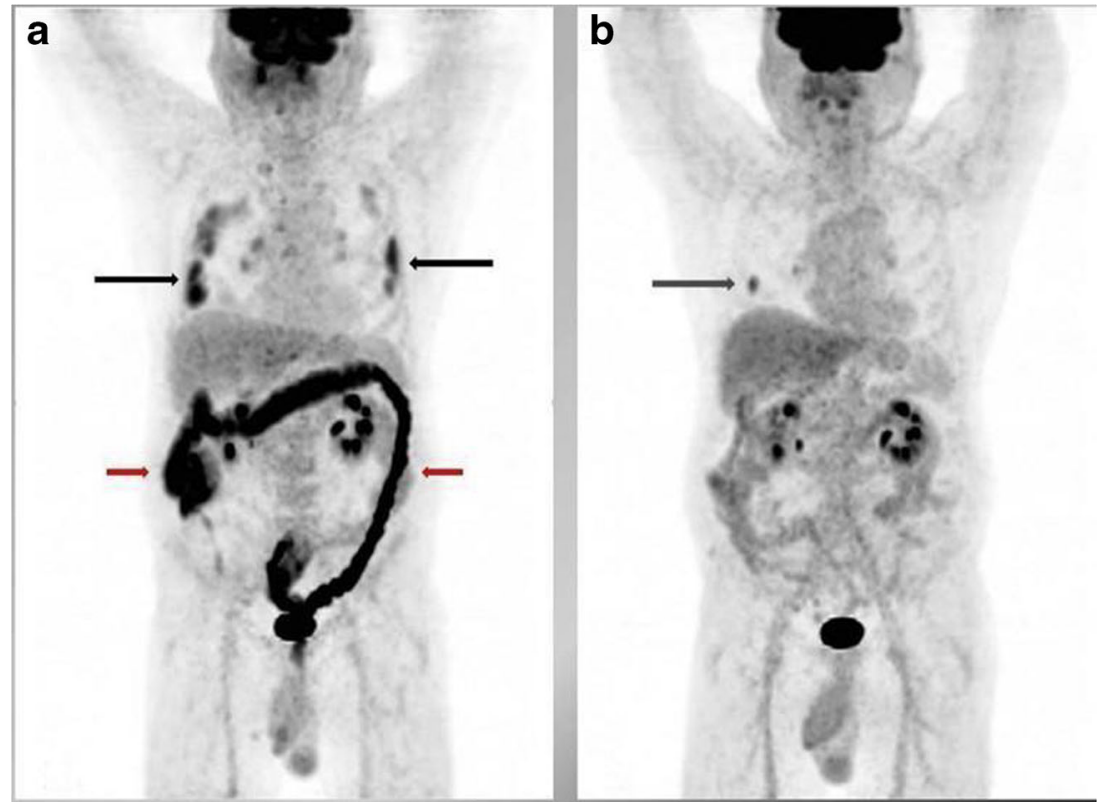


Fig. 3 Delayed imaging for subject 2 (256 MBq injected, 14min scan duration). (Left-to-right) images from scans performed at $1,3,8$, and $10 \mathrm{~h}$ after injection. (Top row) MIP images. (Bottom row) coronal views of thorax and abdomen. Head motion artifacts are visible in 8-h scan (reproduced with permission from Badawi RD et al.) [18]
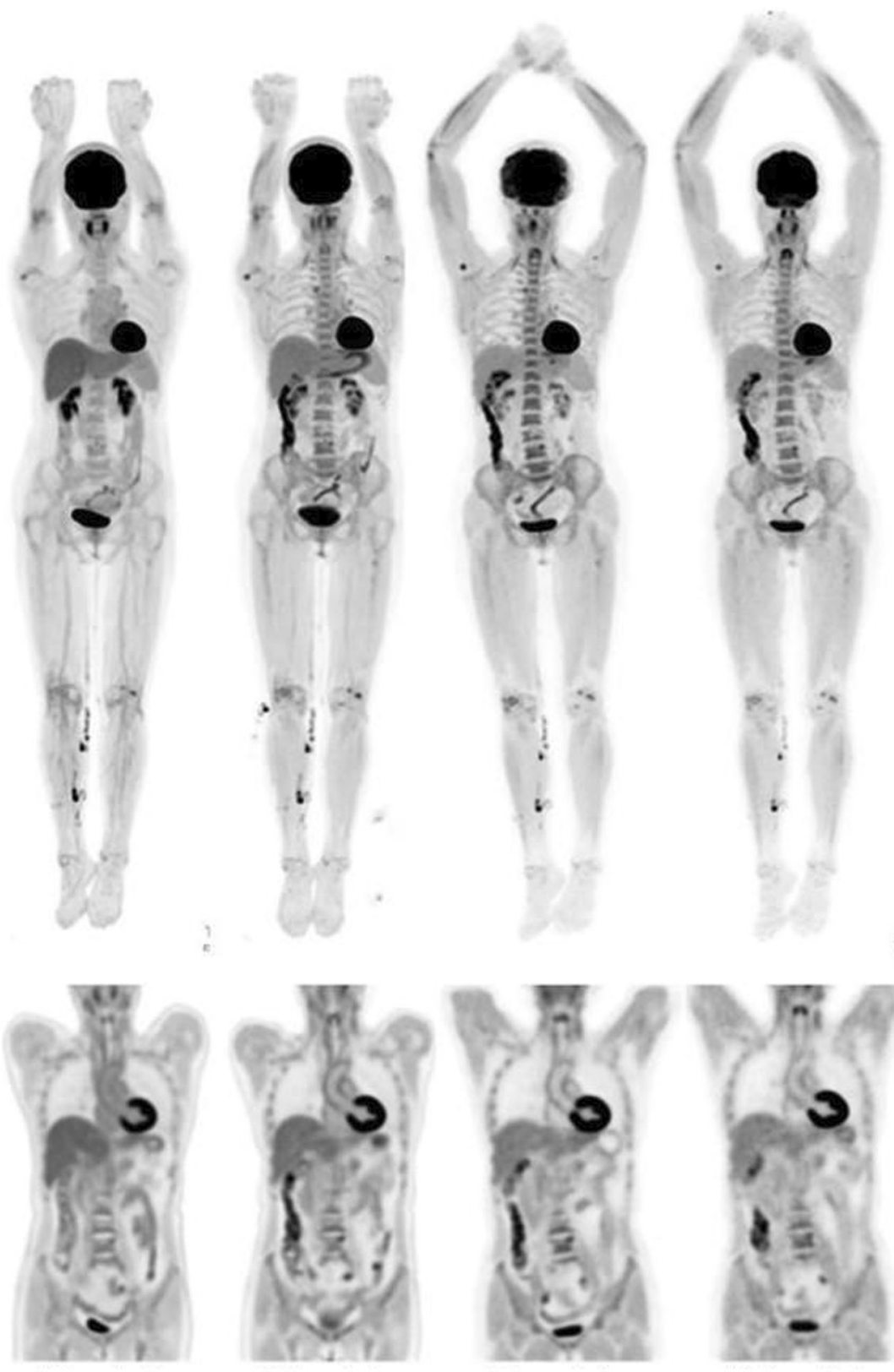

$1 \mathrm{~h}$ uptake
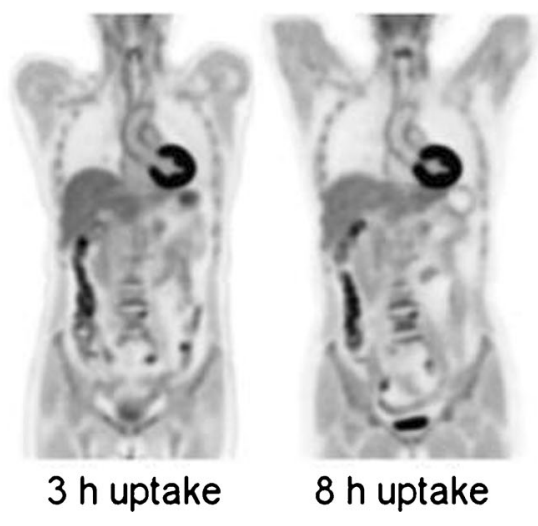

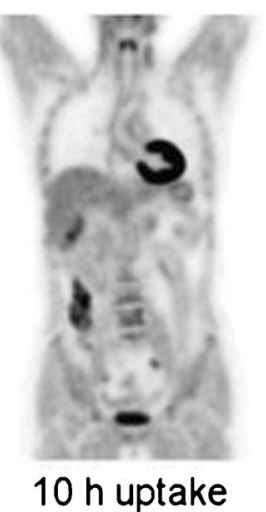

suspected venous thromboembolic disorders in the near future.

Since thromboembolism is considered as one of the major complications of COVID-19 infection, it is likely that total body PET imaging will allow detection of the clots throughout the body in the affected population, leading to early treatment and prevention of pulmonary embolism in these patients. Therefore, we believe total body PET imaging will not only allow detection of pulmonary and extrapulmonary inflammatory process but also visualization of the clots in the venous system in the extremities.
It has been well established that FDG-PET imaging is an effective and sensitive modality for detecting and characterizing various neurological disorders [28]. Because of its high glycolytic activity, the gray matter abnormalities including cerebrovascular accidents are readily detected by FDG-PET imaging. Since patients with COVID-19 infection suffer from such vascular complications, it is likely that this imaging modality will be of great value in assessing cerebrovascular injuries. Also, it is possible that COVID-19-related vasculitis can be detected by FDG-PET imaging [29, 30]. In a recent study, Sollini $M$ et al. evaluated scans of 10 
Fig. 4 Changes in aortic wall and luminal blood FDG activity at different imaging time points as seen on sagittal FDG-PET images of the thoracic aorta. With time, luminal blood activity decreases while the aortic wall activity increases, which improves the arterial wall-to-blood contrast (superior target-to-background ratio) (reproduced with permission from Moghbel M et al.) [22]

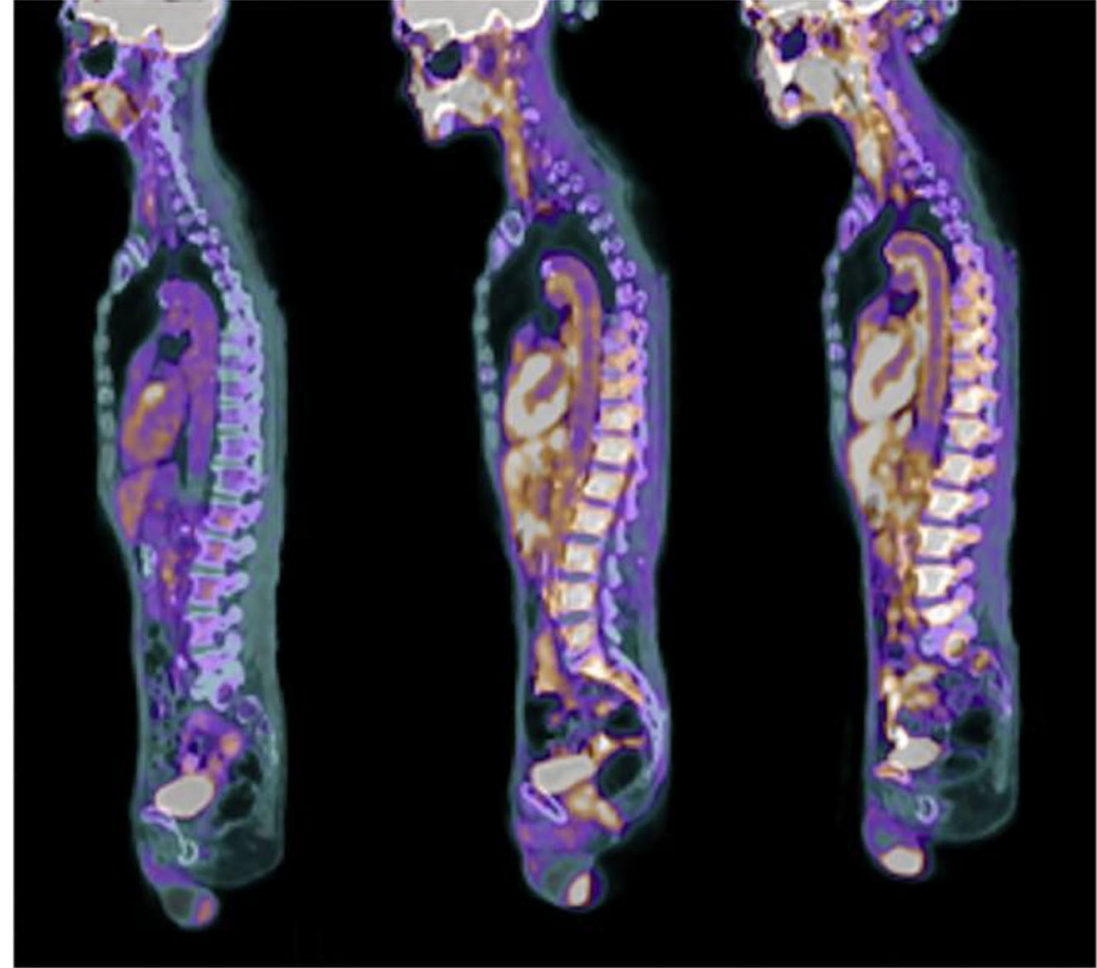

\section{0 minutes 120 minutes 180 minutes}

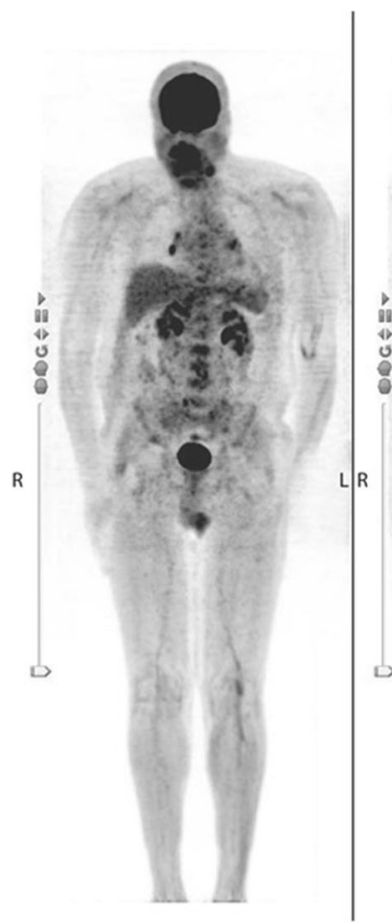

41 d ays after the PECCT

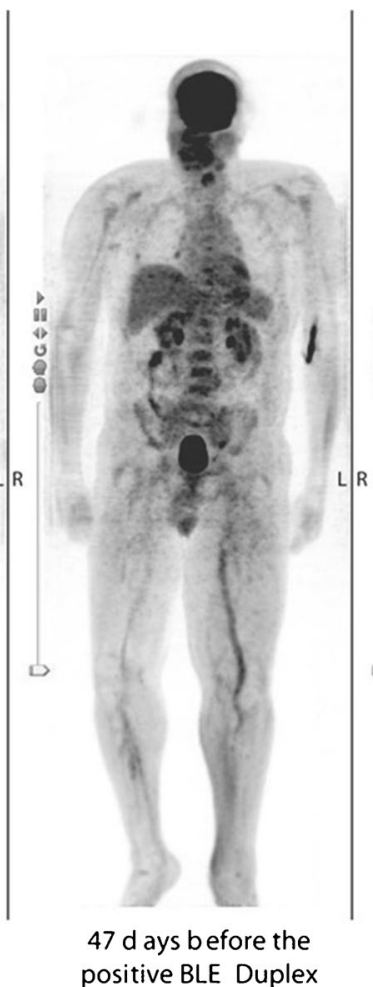

positive BLE Duplex

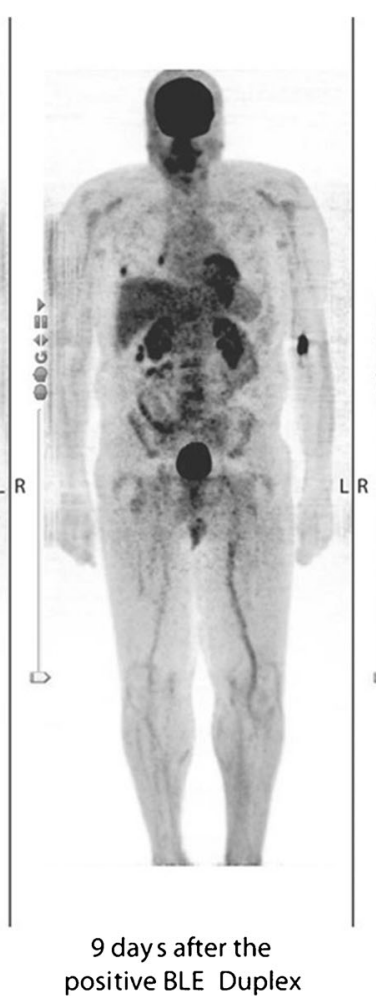

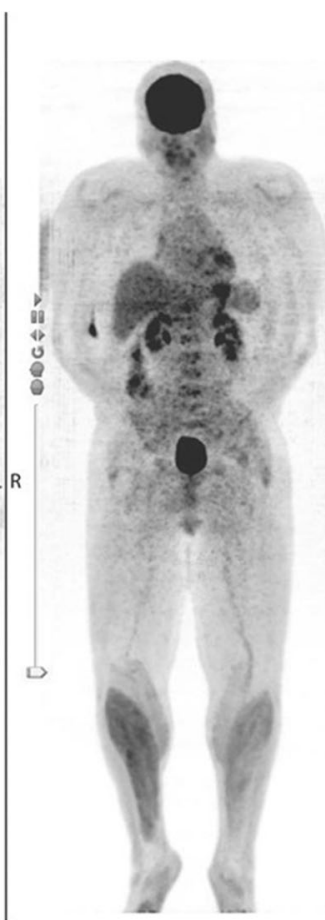

13 d ays before the negative BLE Dup lex

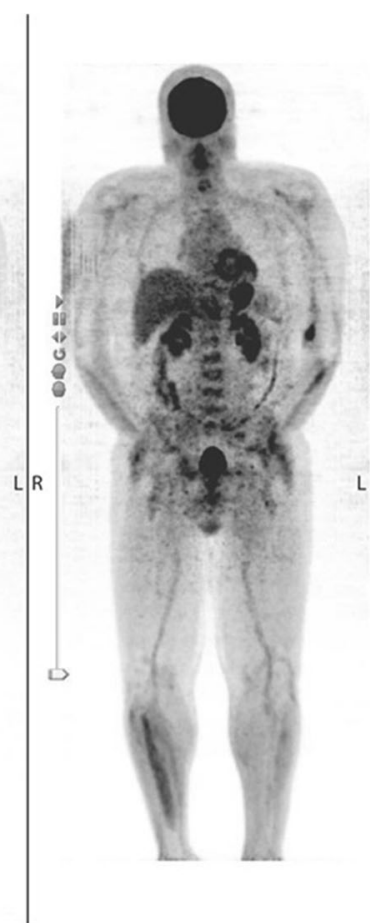

94 d ays after the negative BLE Dup lex
Fig. 5 PECCT initially diagnosed a patient 5 with $\mathrm{PE}$ at the right mainstem pulmonary artery 8 months before the BLE Duplex diagnosed the patient with acute DVT at the left PV. A repeated BLE Duplex 2 months later was negative for acute DVT. From the left to the right are the FDG-PETs 41 days after the PECCT, 47 days before, and 9 days after the positive BLE Duplex, which demonstrate improvement from 47 days before with visualization of bilateral CF and PV only, and
13 days before and 94 days after the negative BLE Duplex for acute DVT, which demonstrate progression of visualization of bilateral CF, PV, left PT, and left peroneal. BLE, bilateral lower extremities; CF, common femoral; DVT, deep vein thrombosis; PE, pulmonary embolism; PECCT, chest computed tomography with PE protocol; PT, posterior tibial; PV, popliteal vein; UE, upper extremity (reproduced with permission from Zhu HJ et al.) [23] 
Fig. 6 FDG-PET/CT in recovered SARS-CoV-2 patients. Example of [18F] FDG bone marrow uptake in a 50-year-old female patient with persisting symptoms (dyspnea and fatigue) lasting for almost 3 months (MIP in a). She took only symptomatic treatment during the acute phase of infection. Example of FDG vascular uptake in a 51-year-old male patient with persisting symptoms (fatigue) lasting for almost 4 months. He developed an acute respiratory distress syndrome, which finally required endotracheal intubation and invasive ventilation. Visually, the FDG uptake was scored as grade 2 at the ascending aorta (a), the aortic $\operatorname{arch}(\mathbf{b})$, and the descending aorta (c) (reproduced with permission from Sollini $\mathrm{M}$ et al.) [30]

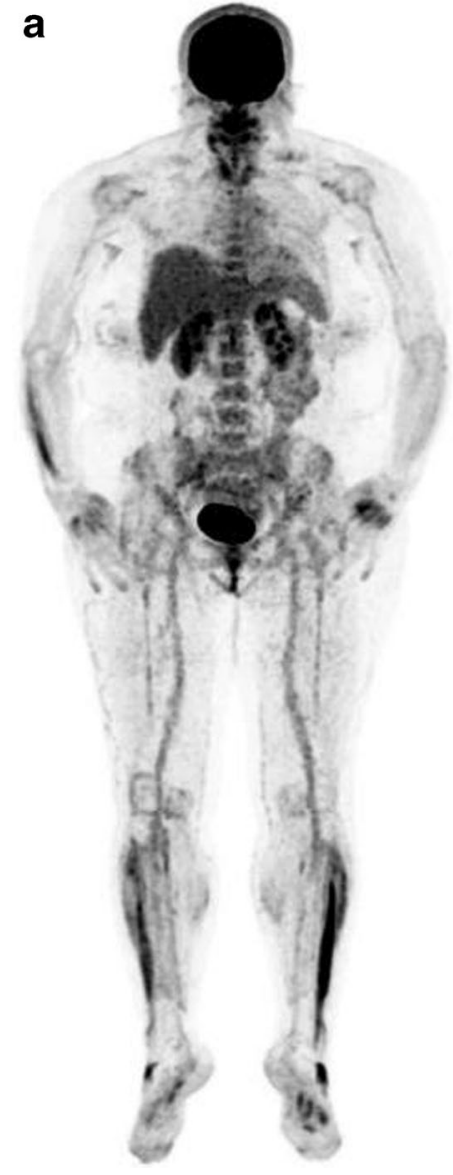

patients who had recovered from COVID-19 and assessed evidence for systemic inflammation by FDG$\mathrm{PET} / \mathrm{CT}$ imaging [30]. These investigators compared the findings in patients with those of control subjects with cancer but with negative follow-up FDG-PET/CT scans. They noted that FDG uptake as measured by target-to-blood pool ratio was significantly higher in COVID-19 patients than in controls in the thoracic aorta, right iliac artery, and femoral arteries (Fig. 6). These data suggest that COVID-19 infection induces vascular inflammation, which can then be measured by FDGPET imaging. FDG-PET is more sensitive than any other competing imaging technique for detecting vascular inflammation. Therefore, PET can provide the opportunity of whole-body screening for inflammatory vascular processes.

One of the major advantages of PET imaging over other imaging modalities is its ability to quantify diffuse disease activity in various organs throughout the body [31-38]. Over the years, we have adopted this technology for global assessment of inflammation in the lungs, atherosclerosis throughout the vascular system, total
C

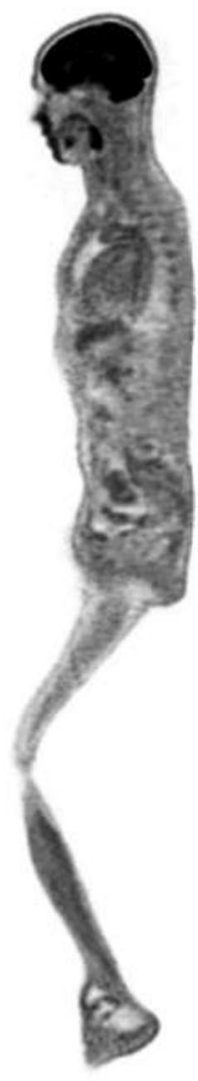

b

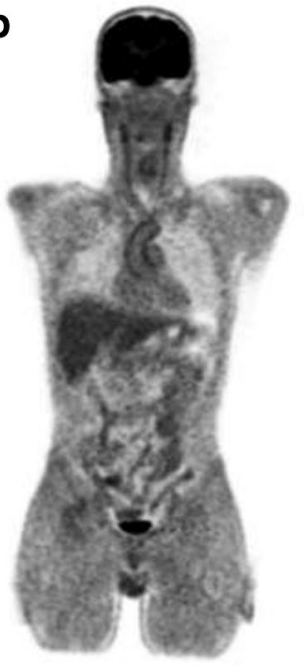

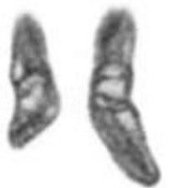

body tumor burden in various malignancies, disorders of the musculoskeletal system, and systemic disorders such as sarcoidosis (Fig. 7) [29]. Therefore, we believe that by performing a whole-body PET scan, we will be able to generate a single number representing the total body burden of the disease and monitor its course following therapeutic interventions [39]. This approach will be particularly of great value in evaluating experimental drugs that are being tested to combat COVID-19 infection. As such, the potential role of total body quantification by this powerful approach appears to be significant and may lead to effective management of the affected population.

In conclusion, we believe that total body FDG-PET imaging of patients with COVID-19 infections will be of great importance for determining the extent of the disease and quantifying its severity in various organs. This will lead to better characterization of this potentially fatal disease and its systemic complication throughout the body. But above all, the unique ability of this methodology to quantify disease activity will allow assessing response to treatment and thereby open new avenues of 
Fig. 7 MIP PET image (a) and fused axial PET images of a sarcoidosis patient show focal increased FDG uptake in supraclavicular, para-aortic, subaortic, paratracheal, and hilar lymph nodes (arrows, $\mathbf{b}-\mathbf{d}$ ) (reproduced with permission from Kung BT et al.) [29]
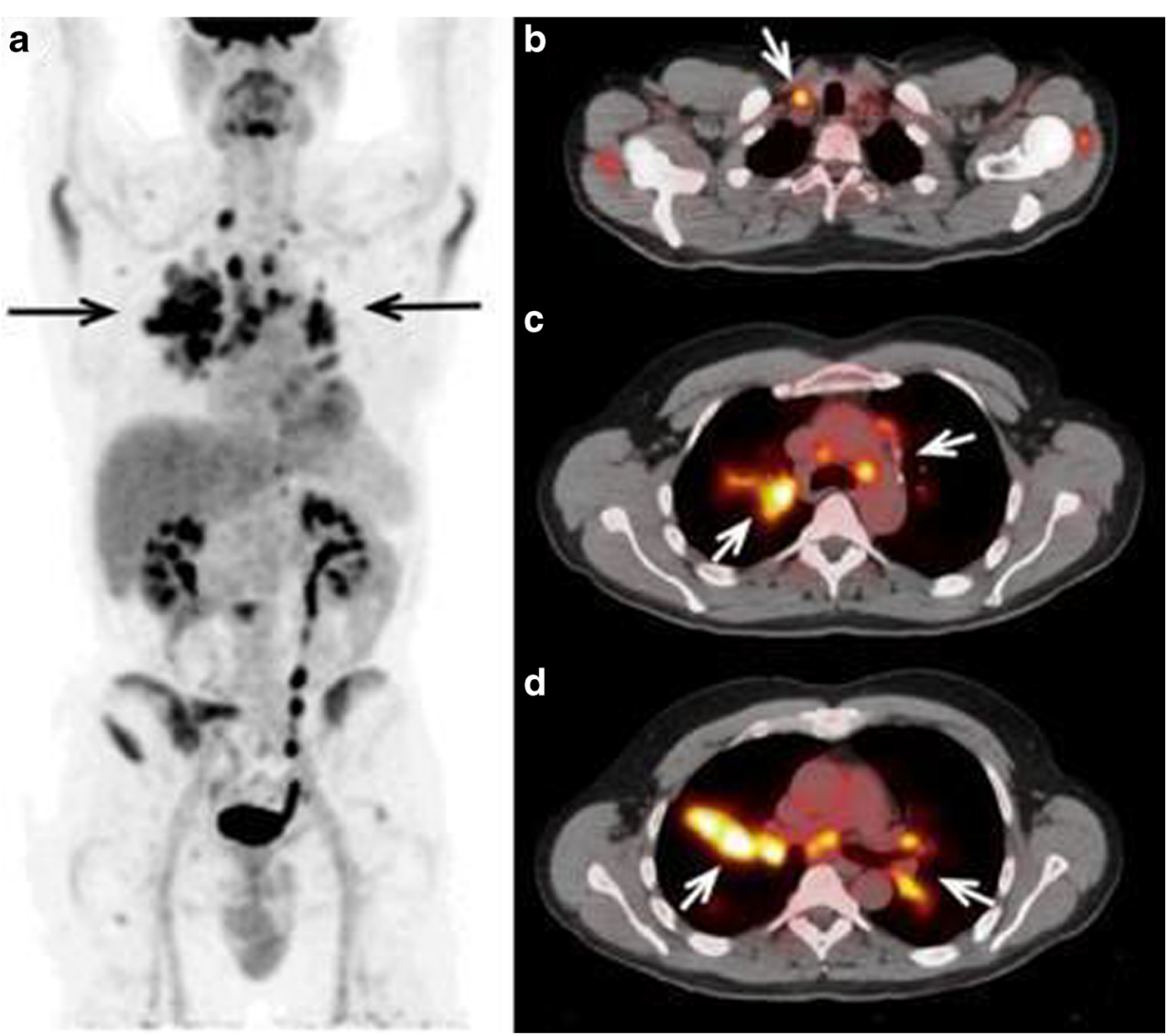

research for drug development for successful management of the affected population.

\section{Compliance with ethical standards}

Conflict of interest The author declares that he has no conflicts of interest.

Ethical approval Institutional Review Board approval was not required because the paper is an Editorial.

\section{References}

1. Behzad S, Aghaghazvini L, Radmard AR, Gholamrezanezhad A. Extrapulmonary manifestations of COVID-19: Radiologic and clinical overview. Clin Imaging. 2020;66:35-41.

2. Grant MC, Geoghegan L, Arbyn M, Mohammed Z, McGuinness L, Clarke EL, et al. The prevalence of symptoms in 24,410 adults infected by the novel coronavirus (SARS-CoV-2; COVID-19): A systematic review and meta-analysis of 148 studies from 9 countries. PLoS One. 2020;15:e234765.

3. Bikdeli B, Madhavan MV, Jimenez D, Chuich T, Dreyfus I, Driggin E, et al. COVID-19 and thrombotic or thromboembolic disease: Implications for prevention, antithrombotic therapy, and follow-up: JACC State-of-the-Art Review. J Am Coll Cardiol. 2020;75:2950-73.

4. Simpson S, Kay FU, Abbara S, Bhalla S, Chung JH, Chung M, et al. Radiological society of North America Expert Consensus
Statement on reporting chest CT findings related to COVID-19. Endorsed by the Society of Thoracic Radiology, the American College of Radiology, and RSNA - Secondary Publication. J Thorac Imaging. 2020;35:219-27.

5. Prokop M, van Everdingen W, van Rees Vellinga T, Quarles van Ufford H, Stöger L, Beenen L, et al. CO-RADS: A categorical CT assessment scheme for patients suspected of having COVID-19definition and evaluation. Radiology. 2020;296:E97-104.

6. Alavi A, Gupta N, Alberini JL, Hickeson M, Adam LE, Bhargava $\mathrm{P}$, et al. Positron emission tomography imaging in nonmalignant thoracic disorders. Semin Nucl Med. 2002;32:293-321.

7. Alavi A, Kung JW, Zhuang H. Implications of PET based molecular imaging on the current and future practice of medicine. Semin Nucl Med. 2004;34:56-69.

8. Basu S, Alavi A. Unparalleled contribution of 18F-FDG PET to medicine over 3 decades. J Nucl Med. 2008;49:17N-21N 37N.

9. Alavi A, Reivich M. Guest editorial: the conception of FDG-PET imaging. Semin Nucl Med. 2002;32:2-5.

10. Czernin J, Phelps ME. Positron emission tomography scanning: Current and future applications. Annu Rev Med. 2002;53:89-112.

11. Hess S, Blomberg BA, Zhu HJ, Hoilund-Carlsen PF, Alavi A. The pivotal role of FDG-PET/CT in modern medicine. Acad Radiol. 2014;21:232-49.

12. Basu S, Chryssikos T, Moghadam-Kia S, Zhuang H, Torigian DA, Alavi A. Positron emission tomography as a diagnostic tool in infection: Present role and future possibilities. Semin Nucl Med. 2009;39:36-51.

13. Basu S, Zhuang H, Torigian DA, Rosenbaum J, Chen W, Alavi A. Functional imaging of inflammatory diseases using nuclear medicine techniques. Semin Nucl Med. 2009;39:124-45.

14. Katal S, Amini H, Gholamrezanezhad A. PET in the diagnostic management of infectious/inflammatory pulmonary pathologies: 
A revisit in the era of COVID-19. Nucl Med Commun. 2020; Available from:. https://doi.org/10.1097/MNM. 0000000000001299

15. Kidane B, Levin DP. Identification and resolution of asymptomatic COVID-19 pneumonitis and colitis: Serial assessment of fluorodeoxyglucose positron emission tomography-computed tomography for evaluation of lung cancer. J Thorac Oncol. 2020; Available from:. https://doi.org/10.1016/j.jtho.2020.09.004.

16. Peleg Y, Kudose S, D'Agati V, Siddall E, Ahmad S, Kisselev S, et al. Acute kidney injury due to collapsing glomerulopathy following COVID-19 infection. Kidney Int Rep. 2020; Available from:. https://doi.org/10.1016/j.ekir.2020.04.017.

17. Minamimoto R, Hotta M, Ishikane M, Inagaki T. FDG-PET/CT images of COVID-19: A comprehensive review. Global Health \& Medicine. jstage.jst.go.jp; 2020;advpub. Available from: https:// doi.org/10.35772/ghm.2020.01056

18. Badawi RD, Shi H, Hu P, Chen S, Xu T, Price PM, et al. First human imaging studies with the EXPLORER total-body PET scanner. J Nucl Med. 2019;60:299-303.

19. Schmall JP, Karp JS, Alavi A. The potential role of total body PET imaging in assessment of atherosclerosis. PET Clin. 2019;14:24550 .

20. Zhang X, Cherry SR, Xie Z, Shi H, Badawi RD, Qi J. Subsecond total-body imaging using ultrasensitive positron emission tomography. Proc Natl Acad Sci U S A. 2020;117:2265-7.

21. Cherry SR, Jones T, Karp JS, Qi J, Moses WW, Badawi RD, et al. Maximizing sensitivity to create new opportunities for clinical research and patient care. J Nucl Med. 2018;59:3-12.

22. Moghbel M, Al-Zaghal A, Werner TJ, Constantinescu CM, Hoilund-Carlsen PF, Alavi A. The role of PET in evaluating atherosclerosis: A critical review. Semin Nucl Med. 2018;48:488-97.

23. Zhu HJ, Hess S, Rubello D, Goris ML, Alavi A. The strong but nonspecific relationship between $18 \mathrm{~F}-\mathrm{FDG}$ uptake in the lowerextremity veins and venous thromboembolism. Nucl Med Commun. 2016;37:322-8.

24. Hess S, Madsen PH, Basu S, Høilund-Carlsen PF, Alavi A. Potential role of FDG PET/CT imaging for assessing venous thromboembolic disorders. Clin Nucl Med. 2012;37:1170-2.

25. Houshmand S, Salavati A, Hess S, Ravina M, Alavi A. The role of molecular imaging in diagnosis of deep vein thrombosis. Am J Nucl Med Mol Imaging. 2014;4:406-25.

26. Kaghazchi F, Borja A, Rojulpote C, Mehdizadeh Seraj S, Zirakchian Zadeh M, Werner T, et al. Role of FDG-PET/CT in the detection and management of venous thromboembolism. J Nucl Med Soc Nuclear Med. 2020;61:1147.

27. Kaghazchi F, Borja A, Mehdizadeh Seraj S, Hancin E, Rojulpote C, Zirakchian Zadeh M, et al. Venous thromboembolism detected by FDG PET/CT in cancer patients: A life-threatening, yet commonly missed observation. J Nucl Med. Soc Nuclear Med. 2020;61:1600.

28. Newberg A, Alavi A, Reivich M. Determination of regional cerebral function with FDG-PET imaging in neuropsychiatric disorders. Semin Nucl Med. 2002;32:13-34.
29. Kung BT, Seraj SM, Zadeh MZ, Rojulpote C, Kothekar E, Ayubcha C, et al. An update on the role of 18F-FDG-PET/CT in major infectious and inflammatory diseases. Am J Nucl Med Mol Imaging. 2019;9:255-73.

30. Sollini M, Ciccarelli M, Cecconi M, Aghemo A, Morelli P, Gelardi F, et al. Vasculitis changes in COVID-19 survivors with persistent symptoms: an [18F]FDG-PET/CT study. Eur J Nucl Med Mol Imaging. 2020; Available from. https://doi.org/10.1007/s00259020-05084-3.

31. Abdulla S, Salavati A, Saboury B, Basu S, Torigian DA, Alavi A. Quantitative assessment of global lung inflammation following radiation therapy using FDG PET/CT: a pilot study. Eur J Nucl Med Mol Imaging. 2014;41:350-6.

32. Alavi A, Newberg AB, Souder E, Berlin JA. Quantitative analysis of PET and MRI data in normal aging and Alzheimer's disease: atrophy weighted total brain metabolism and absolute whole brain metabolism as reliable discriminators. J Nucl Med. 1993;34:16817.

33. Basu S, Saboury B, Werner T, Alavi A. Clinical utility of FDG-PET and $\mathrm{PET} / \mathrm{CT}$ in non-malignant thoracic disorders. Mol Imaging Biol. 2011;13:1051-60.

34. Beheshti M, Saboury B, Mehta NN, Torigian DA, Werner T, Mohler E, et al. Detection and global quantification of cardiovascular molecular calcification by fluoro18-fluoride positron emission tomography/computed tomography-a novel concept. Hell J Nucl Med. 2011;14:114-20.

35. Berkowitz A, Basu S, Srinivas S, Sankaran S, Schuster S, Alavi A. Determination of whole-body metabolic burden as a quantitative measure of disease activity in lymphoma: a novel approach with fluorodeoxyglucose-PET. Nucl Med Commun. 2008;29:521-6.

36. Bural GG, Torigian DA, Chamroonrat W, Alkhawaldeh K, Houseni M, El-Haddad G, et al. Quantitative assessment of the atherosclerotic burden of the aorta by combined FDG-PET and CT image analysis: a new concept. Nucl Med Biol. 2006;33: 1037-43.

37. Mehta NN, Torigian DA, Gelfand JM, Saboury B, Alavi A. Quantification of atherosclerotic plaque activity and vascular inflammation using [18-F] fluorodeoxyglucose positron emission tomography/computed tomography (FDG-PET/CT). J Vis Exp. 2012:e3777.

38. Saboury B, Salavati A, Brothers A, Basu S, Kwee TC, Lam MG, et al. FDG PET/CT in Crohn's disease: Correlation of quantitative FDG PET/CT parameters with clinical and endoscopic surrogate markers of disease activity. Eur J Nucl Med Mol Imaging. 2014;41:605-14.

39. Hoilund-Carlsen PF, Edenbrandt L, Alavi A. Global disease score (GDS) is the name of the game! Eur J Nucl Med Mol Imaging. 2019;46:1768-72.

Publisher's note Springer Nature remains neutral with regard to jurisdictional claims in published maps and institutional affiliations. 